\title{
Graphical Analysis of Capability of a Process Producing a Product Family
}

\author{
M. L. HUANG ${ }^{1}$, K. S. CHEN ${ }^{1, *}$ and R. K. LI $^{2}$ \\ ${ }^{1}$ Department of Industrial Engineering and Management, National Chin-Yi Institute of \\ Technology, Taichung, Taiwan, R. O. C. ${ }^{2}$ Department of Industrial Engineering and \\ Management, National Chiao-Tung University, Hsinchu, Taiwan, R. O. C.
}

\begin{abstract}
Statistical techniques are effective and powerful means of quantifying the variability of processes, analyzing this variability with reference to product requirements, and eliminating this variability in product manufacturing. Many process capability indices have been effectively and widely used to determine whether the quality of a process meets preset targets. However, conventional process capability indices cannot be applied to assess the entire process capability of a product family with nominal-the-best specifications. This work presents a novel process capability index $\left(C_{p p}^{\mathrm{T}}\right)$, which takes into account all family members. The index $C_{p p}$ is a simple transformation from index $C_{p m}$, and $C_{p p}^{T}$ provides additional, individual information concerning the accuracy and precision of a process. Vännman's $(\delta, \gamma)$ plot [Vännman and Deleryd, Quality and Reliability Engineering International 15(3): 213217 (1999)] is revised to compare the process capabilities of family members under both $100 \%$ inspection and sampling plans. Examples are provided to demonstrate the method's practical application.
\end{abstract}

Key words: product family; process capability index; process yield.

\section{Introduction}

In many fields, work done ten years ago is now commonly classified as "obsolete." The very specific field of process capability indices (PCIs) is, in this respect, quite typical, although some early ideas and methods appear to remain important and useful (Kotz and Johnson, 2002). The PCIs have been extensively used to measure the conformation of product quality to requirements in conventional, automotive, semiconductor and IC assembly manufacturing industries. Numerous statisticians and quality engineers, such as Kane (1986), Chan et al. (1988), Choi and Owen (1990), Boyles (1991), Pearn et al. (1992), Kotz and Johnson (1993), Boyles (1994) and

\footnotetext{
${ }^{*}$ Author for Correspondence: K. S. Chen, Department of Industrial Engineering and Management, National Chin-Yi Institute of Technology, Taiwan, 411, R.O.C., E-mail: kschen@ncit.edu.tw.
} 
Spiring (1997), have emphasized the research into process capability indices and proposed more precise methods evaluating process capabilities and performance.

Kane (1986) described six areas of application of capability indices - preventing the production of nonconforming products, measuring continuous improvement, communication, prioritization, identifying directions for improvement, and auditing a quality system. Additionally, Tsui (1997) identified four key aims of PCIs- comparing process performance with specifications, comparing various processes (unitless measures), providing information concerning processes (proportion conforming and/or closeness to target) and specifying directions for quality improvement. These techniques have been successfully implemented. Companies have enjoyed benefits from their use of statistical methods to improve quality and reduce costs. The best example of a successful process capability improvement program is Motorola Inc.'s Six Sigma program. Several capability indices, including $C_{p}, C_{p u}, C_{p l}, C_{p k}$ and $C_{p m}$, have been applied by manufacturing industry as common quantitative measures of process potentials and performance.

$C_{p}$ measures the potential capability of a process, while $C_{p k}$ measures actual capability. According to Boyles (1994), $C_{p}$ and $C_{p k}$ are capability indices with respect to process yield, and are irrelevant to the process target $(T) . C_{p}$ and $C_{p k}$ may fail to account for process centering. Chan et al. (1988) proposed the index $C_{p m}$ that adequately handled process centering. The index $C_{p m}$ clearly models process capability under the loss function approach and adds an additional penalty for being off-target. Considering a process with specifications $U S L=65, L S L=35$, and $T=50$ in Table I (Boyles, 1991), $C_{p k}$ coincides situations A, B with C. Index $C_{p m}$ differentiates among the process shifts from situation $A$ to situations $B$ and $C$, while $C_{p}$ explicates that situations are improving. Obviously, $C_{p m}$ is a better indicator measuring distance from a target.

Greenwich and Jahr-Schaffrath (1995) introduced the index $C_{p p}$ defined as follows:

$$
C_{p p}=\left(\frac{\mu-T}{D}\right)^{2}+\left(\frac{\sigma}{D}\right)^{2},
$$

Table I. Process situations and the corresponding values of $C_{p}, C_{p k}$ and $C_{p m}$

\begin{tabular}{llllll}
\hline Process & $\mu$ & $\sigma$ & $C_{p}$ & $C_{p k}$ & $C_{p m}$ \\
\hline $\mathrm{A}$ & 50.00 & 5.00 & 1.00 & 1.00 & 1.00 \\
$\mathrm{~B}$ & 57.50 & 2.50 & 2.00 & 1.00 & 0.63 \\
$\mathrm{C}$ & 61.25 & 1.25 & 4.00 & 1.00 & 0.44 \\
\hline
\end{tabular}


where $D=d / 3, d=1 / 2(U S L-L S L),(\mu-T)^{2} / D^{2}$ is denoted by inaccuracy index $\left(C_{i a}\right)$ and $(\sigma / D)^{2}$ is denoted by imprecision index $\left(C_{i p}\right)$. Thus, $C_{p p}=C_{i a}+C_{i p}$. The index $C_{p p}$ is a simple transformation from the index $C_{p m}\left(C_{p p}=\left(1 / C_{p m}\right)^{2}\right)$, and $C_{p p}$ provides additional and individual information concerning the process accuracy and the process precision, while this kind of information is unavailable with the use of $C_{p m}$ index.

$$
\text { When } \begin{aligned}
C_{p p} & =\left(\frac{\mu-T}{D}\right)^{2}+\left(\frac{\sigma}{D}\right)^{2} \leq c \\
& \Rightarrow\left(\frac{\mu-T}{D}\right)^{2} \leq c-\left(\frac{\sigma}{D}\right)^{2} \\
& \Rightarrow\left|\frac{\mu-T}{D}\right| \leq \sqrt{c-\frac{\sigma^{2}}{D^{2}}} \leq \sqrt{c} \\
& \Rightarrow \quad T-d \sqrt{c}<\mu<T+d \sqrt{c}
\end{aligned}
$$

Consider a process with specifications $U S L=65, L S L=35$ and $T=50$ in Table II. The $C_{p m}$ index specifies the process capability of processes D, $\mathrm{E}$ and $\mathrm{F}$ all in the same situation. The $C_{p p}$ values for processes $\mathrm{D}, \mathrm{E}$ and $\mathrm{F}$ are all 1.00, that is, the differences in the accuracy and precision among the processes cannot be determined if only the index $C_{p p}$ is applied. Detecting the process inaccuracy and process imprecision using indices $C_{i a}$ and $C_{i p}$ yields a precise direction of quality improvement and enhancement. Thus, $C_{p p}$ is a better choice for engineers who need to measure process potentials and performance. Although $C_{p p}$ is used to evaluate the production of a single product in common situations, $C_{p p}$ cannot be applied to evaluate the integrated process capability for a product family with nominal-the-best specifications.

Accreditation by the Quality System Requirement QS9000 requires an agent to conduct a global evaluation of the process capability over the entire product family from a plant. In practice, suppliers are responsible, obligated and encouraged to provide information on product quality to buyers. Buyers normally book orders for several models within a product family. However, the method of determining whether the integrated

Table II. Process situations and the corresponding values of $C_{p m}, C_{p p}, C_{i a}$ and $C_{i p}$

\begin{tabular}{lllllll}
\hline Process & $\mu$ & $\sigma$ & $C_{p m}$ & $C_{p p}$ & $C_{i a}$ & $C_{i p}$ \\
\hline $\mathrm{D}$ & 50.00 & 5.00 & 1.00 & 1.00 & 0.00 & 1.00 \\
$\mathrm{E}$ & 52.50 & 4.33 & 1.00 & 1.00 & 0.25 & 0.75 \\
$\mathrm{~F}$ & 47.00 & 4.00 & 1.00 & 1.00 & 0.36 & 0.64 \\
\hline
\end{tabular}


process capability of a product family with nominal-the-best specifications meets preset target values to demonstrate a capable process capability has not been explored. Relevant research is limited. Chen et al. (2002a) and Chen et al. (2002b) have discussed the entire process capability for smallerthe-better and larger-the-better, respectively, product families. This work extends the application to assess the integrated process capability for a product family with nominal-the-best specifications.

Section 2 describes the relationship between the process capability index $\left(C_{p p}^{\mathrm{T}}\right) C_{p p}$ and the process yield. Section 3 addresses the integrated process capability of a product family using several models. Section 4 discusses applications of the integrated $C_{p p}^{\mathrm{T}}$ of a product family for both $100 \%$ inspection and sampling plans. Finally, Section 5 draws conclusions.

\section{Relationship between Process Capability Index $C_{p p}$ and Process Yield}

The PCIs have been proven to become valuable means and have been widely used in manufacturing industry to assess effectively and efficiently the process capabilities. The $C_{p p}$ index can not only evaluate the process capability, but also easily distinguish the inaccuracies of the process and the imprecision of the process in terms of the process $C_{i a}$ and the process $C_{i p}$, respectively. From the definition of $C_{p p}$, a smaller value of $C_{p p}$ clearly implies a lower departure ratio. Additionally, when quality characteristic is normally distributed and when $C_{p p}=1 / c^{2}$, the formula for the relationship between $C_{p p}$ and the process yield is:

$$
\text { Yield }=\Phi\left[\frac{1+\sqrt{(1 /(3 c))^{2}-(\sigma / d)^{2}}}{(\sigma / d)}\right]+\Phi\left[\frac{1-\sqrt{(1 /(3 c))^{2}-(\sigma / d)^{2}}}{(\sigma / d)}\right]-1
$$

where $(\sigma / d)=h /(30 c)$ and $(\sigma / d) \leq(1 / 3 c)$, $\Phi$ denotes the standard normal cumulative distribution function. As noted by Chen et al. (2001), process capabilities are categorized into five conditions. Table III displays quality conditions and the corresponding $C_{p p}$ and $C_{p m}$ values. Table IV displays the $C_{p p}$ values and the corresponding process yields.

Obviously, a smaller $C_{p p}$ is associated with a higher process yield from Table II. When $C_{i a}=0$, the relationship between $C_{p p}$ and process yield is $p=2 \Phi\left(3 / \sqrt{C_{p p}}\right)-1$; when $C_{i a} \leq 1$, the relationship between $C_{p p}$ and process yield is $p \geq 2 \Phi\left(3 / \sqrt{C_{p p}}\right)-1$. Practitioners are encouraged to pursue smaller values of $C_{p p}$ to ensure the desired or satisfactory process yields. The foregoing analysis in Section 1 clarifies that a smaller value of $C_{p p}$ implies a lower departure ratio from the target. Additionally, given the formula $C_{p p}=C_{i a}+C_{i p}$, the separate information of the process inaccuracy and the process imprecision give practitioners a better understanding of the 
Table III. The five quality conditions

\begin{tabular}{lll}
\hline Quality condition & $C_{p m}$ & $C_{p p}$ \\
\hline Inadequate & $C_{p m}<1.00$ & $1.00<C_{p p}$ \\
Capable & $1.00 \leq C_{p m}<1.33$ & $0.56<C_{p p} \leq 1.00$ \\
Satisfactory & $1.33 \leq C_{p m}<1.50$ & $0.44<C_{p p} \leq 0.56$ \\
Excellent & $1.50 \leq C_{p m}<2.00$ & $0.25<C_{p p} \leq 0.44$ \\
Super & $2.00 \leq C_{p m}$ & $C_{p p} \leq 0.25$ \\
\hline
\end{tabular}

Table IV. $c$ values and the corresponding process yields $\left(C_{p p}=1 / c^{2}\right)$

\begin{tabular}{llll}
\hline$c$ & $h=1$ & $h=5$ & $h=10$ \\
\hline 1.0 & 1.00000000000000 & 0.99999013608781 & 0.99730020393674 \\
1.1 & 1.00000000000000 & 0.99999943618845 & 0.99903315171523 \\
1.2 & 1.00000000000000 & 0.99999997723638 & 0.99968178281969 \\
1.3 & 1.00000000000000 & 0.99999999935223 & 0.99990380731197 \\
1.4 & 1.00000000000000 & 0.99999999998703 & 0.99997330850197 \\
1.5 & 1.00000000000000 & 0.99999999999982 & 0.99999320465375 \\
1.6 & 1.00000000000000 & 1.00000000000000 & 0.99999841334370 \\
1.7 & 1.00000000000000 & 1.00000000000000 & 0.99999966034652 \\
1.8 & 1.00000000000000 & 1.00000000000000 & 0.99999993335910 \\
1.9 & 1.00000000000000 & 1.00000000000000 & 0.99999998801926 \\
2.0 & 1.00000000000000 & 1.00000000000000 & 0.99999999802682 \\
\hline
\end{tabular}

process and practically provides chances for elevating process capability to a preset target.

\section{Product Family}

For a product family of $k$ product models, the total number $(N)$ of produced products is the sum of the numbers of each product model $\left(N_{i}\right): N=$ $\sum_{i=1}^{k} N_{i}$. The weighting factor for each product model is $w_{i}=N_{i} / N$, and the sum of weighting factors equals $1\left(\sum_{i=1}^{k} w_{i}=1\right)$. If the individual process yield is $p_{i}$ and $N_{i}$ products of model $i$ are produced, the total process yield for the product family is the weighted average of the process yield over $k$ family members. Consequently, the total process yield of the product family is the weighted sum of family members in the product family; that is, $p=\sum_{i=1}^{k} w_{i} p_{i}$.

Index $C_{p p i}$ specifies the $\left(C_{p p}^{\mathrm{T}}\right)$ for product model $i$. The integrated $C_{p p}^{\mathrm{T}}$ is defined as the worst case of $k$ family members; namely, the maximal process capability among family members. Conversely, if the integrated process 
capability is $v$, then any individual process capability of a family member will be smaller than or equal to $v$. The connection between the integrated process capability and the process yield for a product family with $k$ family members is as follows.

$$
\text { When } C_{p p}^{\mathrm{T}}=\max \left\{C_{p p 1}, C_{p p 2}, \ldots, C_{p p k}\right\}=v, C_{p p i} \leq v, \quad i=1,2, \ldots, k \text {. }
$$

When $C_{p p}^{\mathrm{T}} \leq 1$, the total process yield $p=\sum_{i=1}^{k} w_{i} p_{i} \geq \sum_{i=1}^{k} w_{i}[2 \Phi(3 / \sqrt{v})-$ $1]=2 \Phi(3 / \sqrt{v})-1$, yielding $p \geq 2 \Phi(3 / \sqrt{v})-1$. If the integrated process capability of the product family, which equals the worst process capability of all product models, is obtained, then the total process yield is ensured. For example, if the integrated process capability of the product family is 1.0 , the entire process yield is guaranteed to be greater than 0.9973. Consider a product family with four models, and process capabilities for models I-IV are 1.00, 0.56, 0.44 and 0.25 , respectively. Table V lists the corresponding process yields. In that case, the product capability for an entire product family equals the maximum among four models, $C_{p p}^{\mathrm{T}}=1.00$, and the entire process yield exceeds 0.9973 .

\section{Application of the Entire Process Capability Index $C_{p p}^{\mathrm{T}}$}

Three approaches are commonly used to lot sentencing: (1) accept with no inspection; (2) perform a 100\% inspection, and (3) perform acceptance sampling. The no-inspection alternative is used only when the vendor's product is excellent and no defective product is found in the lots. One hundred percent inspection and acceptance sampling are employed most often. These two schemes are comprehensively discussed and examples are presented below.

\subsection{ONE HUNDERED PERCENT INSPECTION}

One hundered percent inspection is typically used in situations in which a component is critical and passing any defectives would result in an unacceptably high cost of failure in subsequent stages, or where the vendor's

Table $V . C_{p p}$ and the corresponding process yield

\begin{tabular}{rll}
\hline Model & Value of $C_{p p}$ & Corresponding yield \\
\hline I & 1.00 & 0.997300 \\
II & 0.69 & 0.999682 \\
III & 0.51 & 0.999973 \\
IV & 0.39 & 0.999998 \\
\hline
\end{tabular}


Table VI. The transformed data for different models of a product family (100\% inspection)

\begin{tabular}{lccc}
\hline Product Model & $\mathrm{Y}$ & $\mu_{y}$ & $\sigma_{y}$ \\
\hline 1 & $Y_{11}, Y_{12}, \ldots, Y_{1 n_{1}}$ & $\mu_{y_{1}}$ & $\sigma_{y_{1}}$ \\
$\vdots$ & $\vdots$ & $\vdots$ & $\vdots$ \\
$i$ & $Y_{i 1}, Y_{i 2}, \ldots, Y_{i n_{i}}$ & $\mu_{y_{i}}$ & $\sigma_{y_{i}}$ \\
$\vdots$ & $\vdots$ & $\vdots$ & $\vdots$ \\
$k$ & $Y_{k 1}, Y_{k 2}, \ldots, Y_{k n_{k}}$ & $\mu_{y_{k}}$ & $\sigma_{y_{k}}$ \\
\hline
\end{tabular}

process capability does not suffice to meet specifications. Automatic inspection equipment is used in screening components to ensure that they conform to specifications in numerous areas of manufacturing. The application of the entire capability index $C_{p p}^{\mathrm{T}}$ is discussed below.

Vännman and Deleryd (1999) introduced a process capability plot to define the capability of the process, called the $(\delta, \gamma)$-plot, where $\delta=(\mu-$ $T) / d$ and $\gamma=\sigma / d$. The $(\delta, \gamma)$-plot is an effective graphical method for theoretically comparing and contrasting different PCIs and it is invariable with respect to the specification limits. Hence, the revised $(\delta, \gamma)$-plot can be applied to compare the process capabilities of different models of a product family. Based on the $(\delta, \gamma)$-plot, the screening data can be compared for different models of a product family according to $Y=(X-T) / d$. The population parameters become

$$
\begin{aligned}
\mu_{y} & =\frac{\mu_{x}-T}{d}, \\
\sigma_{y} & =\frac{\sigma_{x}}{d} .
\end{aligned}
$$

The formula transforms the original specifications from ( $L S L, T, U S L)$ to $(-1,0,1)$. Since $D=d / 3, C_{i a}=(\mu-T)^{2} / D^{2}=9 \mu_{y}^{2}$ and $C_{i p}=(\sigma / D)^{2}=$ $9 \sigma_{y}^{2} \cdot C_{p p}=9\left(\mu_{y}^{2}+\sigma_{y}^{2}\right)$ is achieved. The original $C_{p p}$ is also related to Taguchi loss function expectation, and $C_{p p}$ reasonably reflects the expected loss of the product. Furthermore, the relationship between $C_{p p}$ and the process yield, mentioned in Section 2, reveals the vital connection. Table VI displays the screening and transformed data for different models of a product family. The entire process capability of the product family $\left(C_{p p}^{\mathrm{T}}\right)$ equals the maximal process capability among models.

Unlike $\delta$ and $\gamma$ in the $(\delta, \gamma)$-plot, $\mu_{y}$ represents the $X$-axis and $\sigma_{y}$ represents the $Y$-axis in the $\left(\mu_{y}, \sigma_{y}\right)$-plot.

A smaller $C_{p p}$ value implies better process capability, and the process capability is categorized as inadequate if $C_{p p}$ exceeds 1 . Five process 
capability values exist for five models of a product family in Figure 1. The entire process capability of the product family equals the maximal value among family members; that is, $C_{p p}^{\mathrm{T}}$ equals the process capability of model Q4 and the entire process is considered as incapable. Distance $r_{i}$ is measured and defined as $r_{i}=\sqrt{\mu_{y_{i}}^{2}+\sigma_{y_{i}}^{2}}=(1 / 3) \sqrt{C_{p p i}}$ to make multiplecomparisons of the capabilities of family members. A larger distance $r_{i}$ represents larger capability index, which implies poorer process capability. The magnitudes of $r_{i}$ are arranged in increasing order, and the $i$ th magnitude is then denoted as $r_{(i)}$. From Figure $1, r_{(i)}$ for $i=1$ to 5 represents the magnitudes in ascending order for models Q1, Q5, Q3, Q2 and Q4, respectively. Clearly, the product capability of model Q1 with the smallest value $r_{1}$ is the best. This study aims to distinguish which family members satisfy the requirements, and which family members must be monitored to improve their product capabilities. Performing multiple-comparisons for family members is not the central aim of this research. Fortunately, the multi-model process capability analysis plot can separate inferior product members from the others (Figure 2).

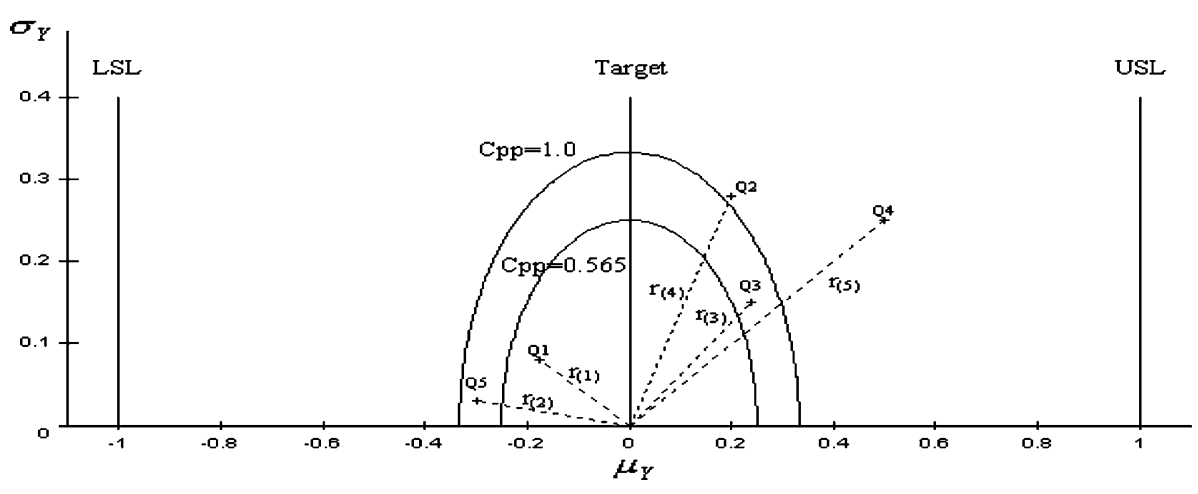

Figure 1. Multi-model process capability analysis plot (I).

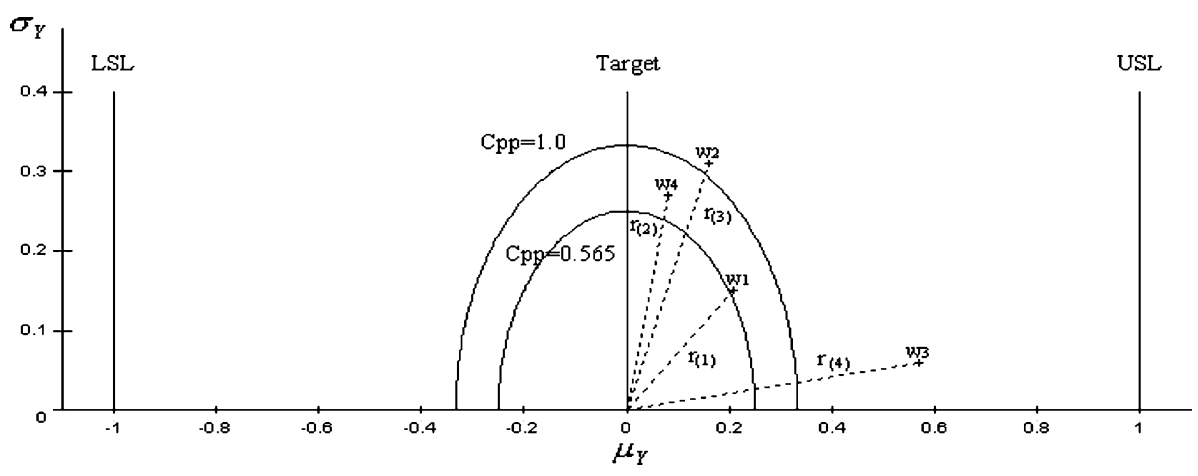

Figure 2. Multi-model process capability analysis plot (II). 
Table VII. Production specifications of as-cut wafers

\begin{tabular}{lllllllll}
\hline Model & $\begin{array}{l}\text { Diameter } \\
(\mathrm{mm})\end{array}$ & $\begin{array}{l}\text { Thickness } \\
(\mathrm{mm})\end{array}$ & $\begin{array}{l}\text { Tolerance } \\
(\mathrm{mm})\end{array}$ & $\mu_{y}$ & $\sigma_{y}$ & $C_{p p}$ & $r$ & Remark \\
\hline $\mathrm{W} 1$ & 76.2 & 300 & \pm 15 & 0.21 & 0.15 & 0.5994 & 0.258 & $r_{(1)}$ \\
W2 & 100 & 320 & \pm 15 & 0.16 & 0.31 & 1.0953 & 0.349 & $r_{(3)}$ \\
W3 & 125 & 400 & \pm 15 & 0.57 & 0.06 & 2.9565 & 0.573 & $r_{(4)}$ \\
W4 & 150 & 700 & \pm 15 & 0.08 & 0.27 & 0.7137 & 0.282 & $r_{(2)}$ \\
\hline
\end{tabular}

The details are as follows.

(i) The process quality of model Q1, which exhibits the best process capability of all product models, greatly surpasses the preset requirement and cheaper materials with acceptable function are considered to be useable to reduce the cost of the product.

(ii) Model Q2 is on the line, $C_{p p}=1.0$. Although the process capability is on the limit, the process must still be monitored to avoid future outof-control situation.

(iii) Models Q3 and Q5 are satisfactory, where $C_{p p}$ is below 1.0. In particular, for model Q5 with a small process variance, a quality enhancement plan is deployed to shift process on-target to increase the process capability when the cost of improvement is acceptable.

(iv) Model Q4 is incapable in terms of both precision and accuracy. Stringent quality enhancement plans are required to find out all assignable causes to reduce process variance and shift process on-target to improve the process capability.

The foregoing evaluation method is applied to determine entire capability for a product family of "as-cut wafers" with diameters of 76.2, 100, 125 and 150. As-cut wafers are sawn from the ingot, without any further processing. The as-cut wafers have very high surface recombination velocities (effectively infinite), so the surfaces must be etched and passivated before meaningful measurements of a product's lifetime can be taken. As-cut wafers are destined for the manufacturing of solar cells in the photomechanical industry, and to manufacture power devices and as test wafers in the electronics industry. Table VII lists the production specifications of as-cut wafers from various, hard materials for various electronic applications. Obviously, the capability of model $\mathrm{W} 1$ with the smallest value of $\hat{C}_{p p}\left(\right.$ denoted by $\left.r_{(1)}\right)$ surpasses the capabilities of the other models.

\subsection{ACCEPTANCE SAMPLING}

Instead of $100 \%$ inspection, acceptance sampling is preferred when testing is destructive, testing cost is extremely high, the vendor's history of quality 
is excellent, or the potential product liability risks are serious, among other circumstances. Practically, determining whether the entire process capability of a product family meets a preset target, to demonstrate a capable process capability, is essential. However, simply examining the $C_{p p}^{\mathrm{T}}$ value, obtained from the sample data, and then making a decision upon whether the process of the entire product family is capable, is extremely unreliable. Therefore, a simple procedure is required for engineers to determine efficiently and correctively whether their processes meet requirements. Here Vännman's $(\delta, \gamma)$-plot (1999) is applied to solve this problem. The confidence intervals of the sample data for each family member are labeled on the plot to reveal the status of each process capability. The entire process capability of the product family equals the maximal process capability among family members.

The index $C_{p p}$ is a function of $\mu_{y}$ and $\sigma_{y}$, where $\mu_{y}$ and $\sigma_{y}$ are unknown. Index $C_{p p}$ is an unknown parameter and should be estimated from a random sample. Hence, the estimator $\hat{C}_{p p}$ is a random variable.

Index $\hat{C}_{p p}$ is defined as

$$
\hat{C}_{p p}=\frac{(\bar{Y}-T)^{2}}{D^{2}}+\frac{S_{Y}^{2}}{D^{2}},
$$

where $D=d / 3, \bar{Y}=\sum_{i=1}^{n} Y_{i} / n$, and $s_{y}^{2}=\sum_{i=1}^{n}\left(Y_{i}-\bar{Y}\right)^{2} /(n-1)$.

Chen (1998) has derived the probability density function of the process capability index $\hat{C}_{p p}$, and the $r$ th moment is defined as follows.

$$
\mathrm{E}\left(\hat{C}_{p p}\right)^{r}=\left(\frac{\sigma^{2}}{n D^{2}}\right)^{r} \sum_{j=0}^{\infty} P_{j}(\lambda) \times \frac{2^{r} \Gamma[(n / 2)+j+r]}{\Gamma[(n / 2)+j]} .
$$

Especially, when $r=1$ and $r=2$, the expected value and the variance become

$$
\begin{aligned}
\mathrm{E}\left(\hat{C}_{p p}\right) & =C_{p p}, \\
\operatorname{Var}\left(\hat{C}_{p p}\right) & =\frac{2 \sigma^{4}}{n D^{4}}+\frac{4(\mu-T)^{2} \sigma^{2}}{n D^{4}} .
\end{aligned}
$$

The transformed formulas for sampling data are similar to those applied in $100 \%$ inspection: $\hat{\mu}_{y}=\bar{Y}$ and $\hat{\sigma}_{y}=s_{y} / c_{4}$. Consider $k$ models in a product family and $n_{i}$ inspected samples of model $i$. Then, $\bar{Y}_{i}=\left(\sum_{j=1}^{n_{i}} Y_{i j}\right) / n_{i}$ and $s_{y_{i}}=\left[\sum_{j=1}^{n_{i}}\left(Y_{i j}-\bar{Y}_{i}\right) /\left(n_{i}-1\right)\right]^{1 / 2}$ are used to estimate the population mean $\mu_{i}$ and the population standard deviation $\sigma_{i}$, respectively. The unbiased estimators for $\mu_{y}$ and $\sigma_{y}$ are $\hat{\mu}_{y}$ and $\hat{\sigma}_{y}$, respectively. The factor $c_{4}=\sqrt{2 /(n-1)} \Gamma[n / 2] / \Gamma[(n-1) / 2]$ is a function of the sample size $n ; c_{4}$ approaches 1, when the sample is sufficiently large. Under the assumption 
Table VIII. The transformed data for different models of a product family (sampling)

\begin{tabular}{lccc}
\hline Product model & $\mathrm{Y}$ & $\hat{\mu}_{y}$ & $\hat{\sigma}_{y}$ \\
\hline 1 & $Y_{11}, Y_{12}, \ldots, Y_{1 n_{1}}$ & $\bar{Y}_{1}$ & $s_{y_{1}} / c_{4}$ \\
$\vdots$ & $\vdots$ & $\vdots$ & $\vdots$ \\
$i$ & $Y_{i 1}, Y_{i 2}, \ldots, Y_{i n_{i}}$ & $\bar{Y}_{i}$ & $s_{y_{i}} / c_{4}$ \\
$\vdots$ & $\vdots$ & $\vdots$ & $\vdots$ \\
$k$ & $Y_{k 1}, Y_{k 2}, \ldots, Y_{k n_{k}}$ & $\bar{Y}_{k}$ & $s_{y_{k}} / c_{4}$ \\
\hline
\end{tabular}

of normality, clearly $\hat{\mu}_{y}$ is normally distributed with a mean of $\mu_{y}$ and a variance of $\sigma_{y}^{2} / n$. $(n-1)\left[\left(c_{4} \hat{\sigma}_{y}\right) / \sigma_{y}\right]^{2}$ follows the $\chi^{2}$ distribution with $(n-1)$ degrees of freedom. Table VIII displays the transformed data for different models of a product family under sampling plans.

The confidence intervals for $\mu_{y}$ and $\sigma_{y}$ are:

$$
\begin{aligned}
& \mu_{y}:\left[\hat{\mu}_{y}-t_{\alpha / 4,(n-1)} \times c_{4} \times \frac{\hat{\sigma}_{y}}{\sqrt{n}}, \hat{\mu}_{y}+t_{\alpha / 4},{ }_{(n-1)} \times c_{4} \times \frac{\hat{\sigma}_{y}}{\sqrt{n}}\right] \\
& \sigma_{y}:\left[\sqrt{\frac{(n-1) \times c_{4}^{2} \times \hat{\sigma}_{y}^{2}}{\chi_{1-\alpha / 4,(n-1)}^{2}}}, \sqrt{\frac{(n-1) \times c_{4}^{2} \times \hat{\sigma}_{y}^{2}}{\chi_{\alpha / 4,(n-1)}^{2}}}\right],
\end{aligned}
$$

where $t_{\alpha / 4,(n-1)}$ is the upper quartile of the $t$ distribution with $(n-1)$ degrees of freedom; $\chi_{1-\alpha / 4,(n-1)}^{2}$ and $\chi_{\alpha / 4,(n-1)}^{2}$ are the upper $(1-\alpha / 4)$ and $(\alpha / 4)$ percentile of the $\chi^{2}$ distribution with $(n-1)$ degrees of freedom. The joint confidence intervals for $\mu_{y}$ and $\sigma_{y}$ are applied to reveal the process situation for each model. The four coordinates of a confidence region for the $\left(\mu_{y}, \sigma_{y}\right)$ vector from upper-right clockwise to upper-left are:

$$
\begin{aligned}
& \text { Upper-right coordinate: }\left(\hat{\mu}_{y}+t_{\alpha / 4},(n-1) \times c_{4} \times \frac{\hat{\sigma}_{y}}{\sqrt{n}}, \sqrt{\frac{(n-1) \times c_{4}^{2} \times \hat{\sigma}_{y}^{2}}{\chi_{\alpha / 4}^{2}(n-1)}}\right), \\
& \text { Bottom-right coordinate: }\left(\hat{\mu}_{y}+t_{\alpha / 4},(n-1) \times c_{4} \times \frac{\hat{\sigma}_{y}}{\sqrt{n}}, \sqrt{\frac{(n-1) \times c_{4}^{2} \times \hat{\sigma}_{y}^{2}}{\chi_{1-\alpha / 4}^{2}(n-1)}}\right) \text {, } \\
& \text { Bottom-left coordinate: }\left(\hat{\mu}_{y}-t_{\alpha / 4},,_{(n-1)} \times c_{4} \times \frac{\hat{\sigma}_{y}}{\sqrt{n}}, \sqrt{\frac{(n-1) \times c_{4}^{2} \times \hat{\sigma}_{y}^{2}}{\chi_{1-\alpha / 4}^{2}(n-1)}}\right), \\
& \text { Upper-left coordinate: }\left(\hat{\mu}_{y}-t_{\alpha / 4},,_{(n-1)} \times c_{4} \times \frac{\hat{\sigma}_{y}}{\sqrt{n}}, \sqrt{\frac{(n-1) \times c_{4}^{2} \times \hat{\sigma}_{y}^{2}}{\chi_{\alpha / 4}^{2}(n-1)}}\right) .
\end{aligned}
$$

In Figure 3, four process capability values are obtained from sampling plans for four models of a product family. As for 100\% inspection in Figure 1, the entire process capability of the product family equals the maximal value among all the product models. That is, $C_{p p}^{\mathrm{T}}$ equals the process capability of model F4 in this case and the entire process is 


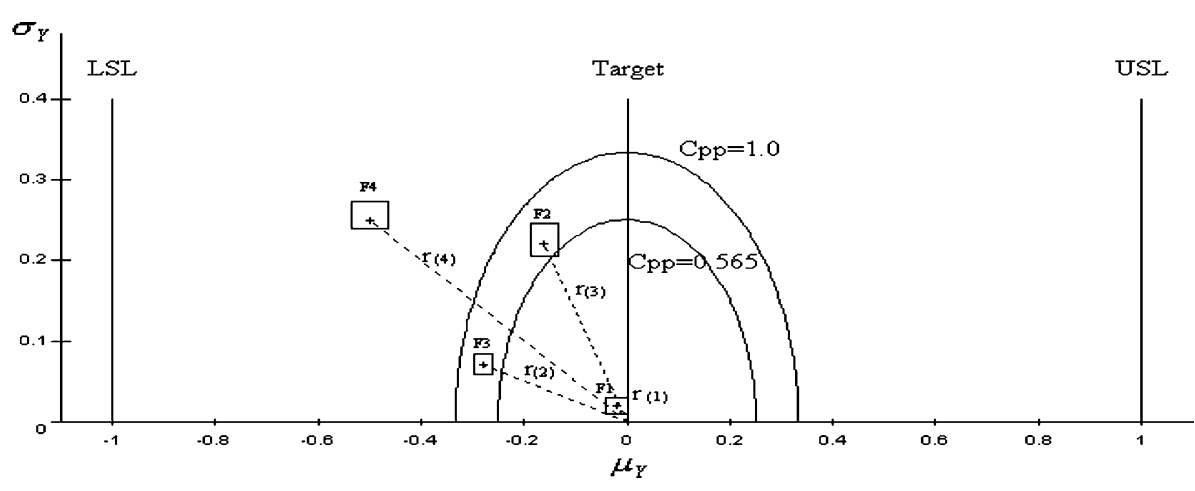

Figure 3. Multi-model process capability analysis plot (III).

categorized as incapable. Distance $r_{i}$ is measured and $r_{(i)}$ is the place of the $i$ th magnitude of distance $r_{i}$ arranged in ascending order, for $i=1$ to 4 to make multiple comparisons of the capacities among family members. Let $\Delta r_{(i)}=r_{(i+1)}-r_{(i)} ; \delta_{i}$ denotes the half diagonal magnitude of the confidence interval for $r_{(i)}$ to determine whether two confidence intervals overlap. Additionally, let

$$
f_{i}=\frac{\delta_{i}+\delta_{i+1}}{\Delta r_{i}}
$$

If $f_{i}<1$, then $r_{(i)}$ is concluded to be shorter than $r_{(i+1)}$. Otherwise, the conclusion is reversed. The process capability for the entire product family is defined as the worst case among the models. Multiple capabilities are presented for the models in a single plot to simplify the multiple-comparisons.

The process situations are as follows.

(i) The process quality of model F1, which exhibits the best process capability among all product models, greatly exceeds the preset quality requirement and cheaper materials, with acceptable function can be used to reduce product cost.

(ii) The joint confidence intervals in model F2 include the value of $C_{p p}=1.0$. Although the process capability includes the limit, the process must still be monitored to avoid a future out-of-control situation.

(iii) Model F3 is in the satisfactory region, where $C_{p p}$ is below 1.0. The process capability is acceptable and no stringent improvement plans need be implemented to enhance the quality.

(iv) Model F4 is incapable, both in terms of precision and accuracy. Immediate quality enhancement plans are required to find out all assignable causes both to reduce the process variance and to shift the process on target and thereby increase the process capability. 
Table IX. Production specifications of Backlight modules

\begin{tabular}{|c|c|c|c|c|c|c|c|c|}
\hline Model & Diameter & $\begin{array}{l}\text { Target } \\
(\mathrm{mm})\end{array}$ & $\begin{array}{l}\text { Tolerance } \\
(\mathrm{mm})\end{array}$ & $\mu_{y}$ & $\sigma_{y}$ & $\hat{C}_{p p}$ & $r$ & Remark \\
\hline B1 & $14 "$ series & 294.4 & \pm 0.15 & 0.12 & 0.05 & 0.1521 & 0.130 & $r_{(1)}$ \\
\hline B2 & $15 "$ series & 315.9 & \pm 0.30 & 0.30 & 0.20 & 1.1700 & 0.361 & $r_{(3)}$ \\
\hline B3 & 17 " series & 366.5 & \pm 0.30 & -0.10 & 0.15 & 0.2925 & 0.180 & $r_{(2)}$ \\
\hline
\end{tabular}

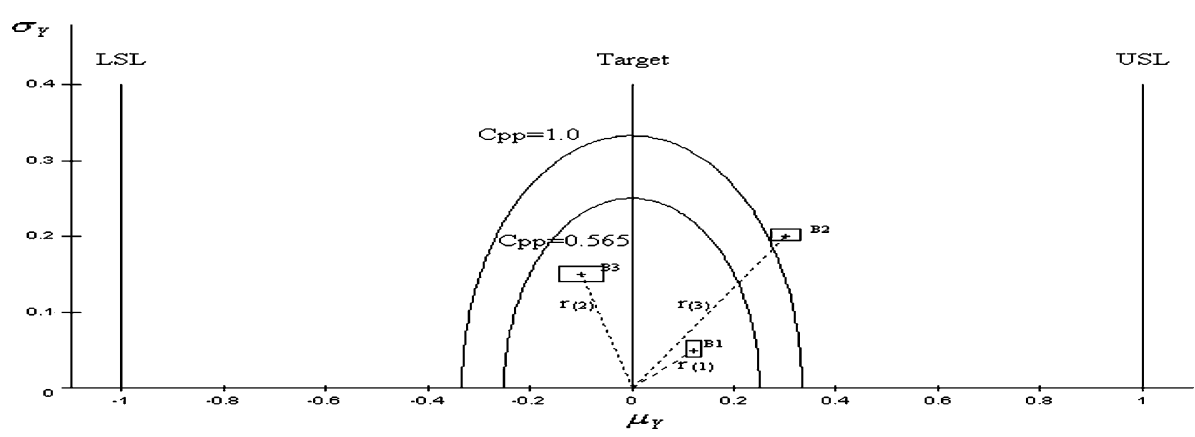

Figure 4. Multi-model process capability analysis plot (IV).

Measuring the entire capability for a product family "backlight module" for size 14-17 inches, the foregoing evaluation method is applied. Initially, backlight module was used primarily in advertising light boxes. However, because of the vigorous marketing of LCD manufacturing and customer demand for night version and full color LCD, backlight module is closely associated with optical products. Generally, backlight module comprises a piece of light-guide plate, which guides a spontaneous light source from a light emitting diode or light tube to create a lager and more uniform surface illuminant. Backlight module is generally simply termed backlight. The application of backlight specializes in supplying the light required by Liquid Crystal. LCD, Hand-phone LCD, and PDA LCD are examples of backlight applications. The advantages of backlight are uniform light distribution and high brightness. Backlight module provides the light source for TFT monitors. Forhouse Corporation is located in central Taiwan and specializes in Cold Cathode Fluorescent Lamp (CCFL) production. TFT monitors are growing in size, and the present production size in Forhouse Corporation ranges from 14 to 17 inches. The detail information regarding the three models is summarized in Table IX. Obviously, the capability of model B1 with the smallest value of $\hat{C}_{p p}$ (denoted by $r_{(1)}$ ) surpasses the capabilities of other two models (Figure 4). 


\section{Conclusions}

High product quality, sound business reputation, superior customer service, reasonable product cost, and other factors are fundamental in retaining customer loyalty. The PCIs, which are widely used to measure whether product quality conforms to requirements, can help companies to promote marketing sales, retain customers and build a company's reputation. This study has overcome the limitations of PCIs used to treat a product family with several product models. The products in a family all have the same function and design; only the sizes differ. Customarily, one product model is measured using one $C_{p p}^{\mathrm{T}}$, but the process capability of the entire product family remains unknown. The $C_{p p}$ index is a simple transformation from index $C_{p m}$, and provides individual information concerning the accuracy and precision of the process. $C_{p p}$ reasonably reflects the expected loss of the product. Additionally, a one-to-one relationship exists between process yield and $C_{p p}$. Vännman's $(\delta, \gamma)$-plot (1999) is revised to compare the process capabilities for different models in a product family. Accordingly, this paper proposes the capability index that considers all members of a product family. For practical applications, evaluation plots are well established and examples are provided to determine whether the process capability of an entire product family meets the preset target for $100 \%$ inspections and sampling plans.

In future research the authors would like to address the notion of "composite reliability" (Chen and Singpurwalla, 1996) and its hierarchical Bayes estimation as a measure of the overall reliability of a collection of heterogeneous but similar items.

\section{Acknowledgements}

The authors would like to thank the National Science Council of the Republic of China for financially supporting this research under Contract No. NSC-90-2213-E-167-002.

\section{References}

Boyles, R. A. (1991). The Taguchi capability index. Journal of Quality Technology 23(1): 1726.

Boyles, R. A. (1994). Process capability with asymmetric tolerances. Communications in Statistics - Simulation and Computation 23(3): 615-643.

Chan, L. K., Cheng, S. W., \& Spiring, F. A. (1988). A new measure of process capability: $C_{p m}$. Journal of Quality Technology 20(3): 162-175.

Chen, K. S. (1998). Estimation of the process incapability index. Communications in Statistics-Theory and methods 27(5): 1263-1274.

Chen, K. S., Chen, S. C. \& Li, R. K. (2002a). Process quality analysis of products. International Journal of Advanced Manufacturing Technology 19(8): 623-628. 
Chen, K. S., Li, R. K., \& Liao, S. J. (2002b). Capability evaluation of a product family for processes of the larger-the-better type. International Journal of Advanced Manufacturing Technology 20(11): 824-832.

Chen, K. S., Huang, M. L., \& Li, R. K. (2001). Process capability analysis for an entire product. International Journal of Production Research 39(17): 4077-4087.

Chen, J. \& Singpurwalla, N. D. (1996). The notion of "composite reliability" and its hierarchical Bayes estimation. Journal of the American Statistical Association 91(436): 1474 1484.

Choi, B. C. \& Owen, D. B. (1990). Applied Multivariate Statistical Analysis, 3rd edn. (New York: Prentice-Hall).

Greenwich, M. \& Jahr-Schaffrath, B. L. (1995). A process incapability index. International Journal of Quality \& Reliability Management 12(4): 58-71.

Kane, V. E. (1986). Process capability indices Journal of Quality Technology 18(1): 41-52.

Kotz, S. \& Johnson, N. L. (1993). Process Capability Indices. London: Chapman and Hall.

Kotz, S. \& Johnson, N. L. (2002). Process capability indices - A review, 1992-2000. Journal of Quality Technology 34(1): 2-53.

Pearn, W. L., Kotz, S. \& Johnson, N. L. (1992). Distributional and inferential properties of process capability indices. Journal of Quality Technology 24(4): 216-231.

Spiring, F. A. (1997). An unifying approach to process capability indices. Journal of Quality Technology. 29(1) 49-58.

Tsui, K. L. (1997). Interpretation of process capability indices and some alternatives. Quality Engineering, 9(4): 587-596.

Vännman, K. \& Deleryd, M. (1999). Process capability plots - A quality improvement tool. Quality and Reliability Engineering International 15(3): 213-217. 